\title{
Structure et dynamique des infracommunautés de Myxosporidies parasites de Ctenopoma petherici GÜNTHER, 1864 (Anabantidae), Clarias pachynema BOULENGER, 1903 (Clariidae) et Hepsetus odoe (BLOCH, 1794) (Hepsetidae) dans la rivière Sangé au Cameroun
}

\author{
Guy Benoît LEKEUFACK FOLEFACK* et Abraham FOMENA
}

Laboratoire de Parasitologie et d'Ecologie, Département de Biologie et Physiologie Animale, Faculté des

Sciences, Université de Yaoundé I, B.P 812 Yaoundé, Cameroun.

*Auteur correspondant, E-mail : leguyzo@yahoo.fr ; Tel : +237 77887294

\section{RÉSUMÉ}

L'examen de Ctenopoma petherici, Clarias pachynema et Hepsetus odoe récoltés dans la rivière Sangé au Cameroun entre janvier 2008 et mars 2009 a révélé la présence de 11 espèces de Myxosporidies. Parmi les espèces parasites identifiées, Myxobolus pethericii et Henneguya pethericii sont apparues fréquentes $(\operatorname{Pr}>$ 50\%); Myxobolus sp.1, Myxobolus sp.2, Myxobolus gariepinus, Myxobolus africanus, Henneguya nkamensis, Myxidium petrocephali et Myxidium nkamensis sont apparues secondaires (10\% $\leq \operatorname{Pr} \leq 50 \%)$, tandis que Myxobolus camerounensis et Myxidium sangei sont apparues rares $(\operatorname{Pr}<10 \%)$. La charge kystique moyenne est apparue élevée ( $\overline{\mathrm{x}}>100$ kystes) pour H. pethericii et H. nkamensis; moyenne (50 kystes $<\overline{\mathrm{x}} \leq 100$ kystes) pour Myxobolus sp.1, Myxobolus sp.2, M. pethericii, M. africanus, et très faible ( $\overline{\mathrm{x}}<10$ kystes) pour $M$. gariepinus. Le pourcentage d'infestation décroît lorsque la taille du poisson augmente pour les espèces parasites suivantes : M. pethericii, M. gariepinus, H. pethericii, H. nkamensis et M. nkamensis. Par ailleurs, la charge kystique moyenne diminue lorsque la taille de l'hôte augmente pour M. pethericii et $H$. pethericii. Hormis le fait que les individus femelles sont fréquemment plus parasités par $H$. pethericii que les mâles, le sexe de l'hôte n'a pas d'influence sur le parasitisme des Myxosporidies étudiées. La saison sèche s'est avérée favorable à l'occurrence de Myxobolus sp.2 alors que M. petrocephali, M. nkamensis et M. gariepinus ont été plus fréquentes en saison de pluies. La charge kystique moyenne de $H$. pethericii et $M$. pethericii est plus importante en saison sèche.

(C) 2013 International Formulae Group. All rights reserved.

Mots clés : Prévalence, charge kystique, poisson, eaux douces.

\section{INTRODUCTION}

Malgré son importance économique et alimentaire, le poisson constitue un biotope très favorable au développement d'un grand nombre de parasites au rang desquels les
Myxosporidies (Lom et Dyková, 2006). Estimée à environ 2180 espèces, les Myxosporidies peuvent être à l'origine de graves épizooties, susceptibles d'affaiblir ou de tuer le poisson hôte, entrainant ainsi des 
pertes économiques importantes (Lom et Dyková, 2006). En recherchant les facteurs susceptibles d'avoir une influence directe sur la faune parasitaire d'un poisson, Renault et Guichard (2007) arrivent à la conclusion selon laquelle dans tout habitat, la faune parasitaire est déterminée par une combinaison de plusieurs facteurs. Parmi ceux-ci, l'âge et le sexe de l'hôte, l'abondance de la nourriture pour l'hôte et la saison, constituent les facteurs les plus importants. Aucun médicament efficace utilisable en pratique dans la lutte contre les myxosporidioses n'est disponible jusqu'à ce jour. Face à un agent potentiellement pathogène émis dans l'environnement de façon constante (Kent et al., 2001), la prévention de la maladie ne peut consister qu'à maintenir l'infection du poisson hôte en deçà du seuil clinique (Mladineo, 2003). Ainsi, il est nécessaire d'étudier les principaux facteurs intrinsèques et extrinsèques modulant la gravité de la myxosporidiose chez les poissons.

En Afrique, les Myxosporidies parasites de poissons d'eaux douces ont déjà fait l'objet de nombreux travaux. Cependant, la plupart de données disponibles portent sur la taxonomie de ces organismes. Les travaux effectués au cours des dix dernières années et qui traitent de la dynamique des populations de Myxosporidies sont ceux de Tombi et Bilong Bilong (2004), Abakar-Ousman et al. (2007), Nchoutpouen et al. (2011). En situation d'élevage, en accentuant la densité et le confinement des hôtes, on modifie les conditions physico-chimiques du milieu qui deviennent plus favorables au déclenchement des épizooties qui peuvent provoquer des mortalités massives de populations de poissons (Boungou et al., 2013). Face à cette situation, Piasecki et al. (2004) soulignent la nécessité de réaliser des études approfondies de taxonomie et de biologie des parasites et/ou des agents pathogènes potentiels en milieu naturel, avant toute tentative de reproduction des hôtes en milieu artificiel.

La présente étude traite de quelques aspects écologiques des Myxosporidies parasites de Ctenopoma petherici (Anabantidae), Clarias pachynema (Clariidae) et Hepsetus odoe (Hepsetidae) capturés dans la rivière Sangé. Ces espèces de poissons sont d'un grand intérêt pour l'alimentation humaine; de plus, Clarias pachynema est impliqué dans des projets de développement piscicole.

\section{MATÉRIEL ET MÉTHODES Milieu d'étude}

Les Poissons examinés ont été récoltés mensuellement de Janvier 2008 à Mars 2009, dans la rivière Sangé (bassin du Wouri) à Ntondé (situé entre le $4^{\circ} 12^{\prime}$ et $4^{\circ} 17^{\prime}$ latitude Nord et le $10^{\circ}$ et $10^{\circ} 8^{\prime}$ longitude Est, Cameroun, Afrique Centrale). Ntondé, situé à $45 \mathrm{~km}$ du fleuve Wouri, est un village de l'Arrondissement de Yabassi (Département du Nkam, Région du Littoral). Le climat à Ntondé est de type équatorial, caractérisé par une forte humidité et deux saisons: une saison sèche de courte durée (Décembre à Février) et une longue saison pluvieuse (Mars à Novembre).

\section{Echantillonnage des poissons}

Les poissons ont été capturés à l'aide de filets maillants puis fixés dans une solution de formol à $10 \%$. Au laboratoire, la position systématique des poissons échantillonnés à été tirées de l'ouvrage de Stiassny et al. (2007). Leur longueur standard a été mesurée au millimètre près. A l'aide de la formule modifiée de Yule (Rioul, 2008), les échantillons ont été regroupés en classes de taille. Le sexe du poisson a été déterminé après identification des gonades. 


\section{Identification de Myxosporidies}

Au laboratoire, les poissons ont d'abord été examinés à l'œil nu, puis à la loupe binoculaire de marque Olympus BO61 en vue de déceler la présence éventuelle des kystes. Après dissection, les organes internes (reins, vésicule biliaire, foie, vessie gazeuse, tube digestif, rate, cœur, gonades, vessie urinaire et urètre) ont été individuellement examinés. Les kystes trouvés ont été dilacérés et leur contenu identifié entre lame et lamelle à l'objectif 100X du microscope. Les spores ont été fixées au méthanol et colorés au May-GrünwaldGiemsa. Les photographies de spores ont été prises à l'aide d'un microscope Olympus $\mathrm{CH}$ 2 équipé d'un dispositif de microphotographie. Les différentes espèces de Myxosporidies ont été identifiées selon les recommandations données par Lom et Arthur (1989).

\section{Indices épidémiologiques}

la prévalence parasitaire (Pr), exprimée en pourcentage, a été calculée en faisant le rapport entre le nombre d'individus d'une espèce hôte infestés par une espèce parasite et le nombre total d'hôtes examinés (Bush et al., 1997).

Se référant à l'intensité moyenne (Bush et al., 1997), la charge kystique moyenne ( $\bar{x})$ a été définie comme la somme des kystes d'une espèce parasite dans un échantillon d'hôtes examinés divisée par le nombre d'hôtes hébergeant au moins un kyste de cette espèce parasite.

\section{Analyse et tests statistiques}

L'analyse du statut de chaque espèce parasite à été faite selon Valtonen et al. (1997). Ainsi, les espèces ont été qualifiées de fréquentes ou communes ou encore principales $(\operatorname{Pr}>50 \%)$, peu fréquentes ou secondaires ou intermédiaires $(10 \% \leq \operatorname{Pr} \leq$ $50 \%)$ et rares ou satellites $(\operatorname{Pr}<10 \%)$. La classification du parasitisme sur la base des charges kystiques moyennes à été faite selon Bilong Bilong et Njiné (1998). Ainsi, la charge kystique moyenne a été qualifiée de très faible si $\overline{\mathrm{x}}<10$; faible si $10 \leq \overline{\mathrm{x}} \leq 50$; moyenne si $50<\overline{\mathrm{x}} \leq 100$ et forte si $\overline{\mathrm{x}}>100$.

La comparaison des pourcentages d'infestation a été faite à l'aide du test de chi $\operatorname{deux}\left(\chi^{2}\right)$. Le test $\mathrm{H}$ de Kruskal-Wallis a permis de comparer plusieurs moyennes alors que le test $U$ de Mann Whitney a permis de comparer les moyennes prises deux à deux. Le coefficient de corrélation " $r$ " de rang de Spearman a été calculé pour rechercher l'existence d'un lien entre différentes variables. Le programme SPSS 12.0 a été utilisé pour tous les tests statistiques et le niveau de sécurité retenu est de $95 \%$.

\section{RÉSULTATS}

Structure des populations de poissons hôtes

$\mathrm{Au}$ total, 863 poissons appartenant à trois espèces ont été récoltés et examinés. Ces espèces de poissons sont: Clarias pachynema (402 individus), Ctenopoma petherici (364) et Hepsetus odoe (97). La longueur standard (LS) de Ctenopoma petherici a varié de 51 à $179 \mathrm{~mm}$, avec une moyenne de 105,18 \pm 21,40 mm. Sur la base de ces longueurs, cette population échantillonnée a été subdivisée en 4 classes de taille, d'amplitude $25 \mathrm{~mm}$. La longueur standard de $H$. odoe a varié de 118 à $260 \mathrm{~mm}$ (longueur moyenne : 177,42 \pm 32,53 $\mathrm{mm}$ ) et 4 classes de taille d'amplitude $35 \mathrm{~mm}$ ont été obtenues. La longueur standard de $C$. pachynema à varié de 55 à $313 \mathrm{~mm}$ et 4 classes de taille d'amplitude $50 \mathrm{~mm}$ ont été obtenues. Pour les trois espèces hôtes, la sexratio est biaisée en faveur des mâles. Elle vaut approximativement 1,4 (211 mâles sur 153 femelles) pour $C$. petherici ; 1,9 (63 mâles sur 34 femelles) pour $H$. odoe et 1,1 (208 mâles sur 194 femelles) pour C. pachynema. 
Inventaire, indices épidémiologiques et statuts des espèces parasites

Dans la rivière Sangé, l'étude de la faune des Myxosporidies parasites de $C$. petherici, $H$. odoe et $C$. pachynema a révélé la présence de 11 espèces appartenant aux genres Myxobolus, Henneguya et Myxidium (Figure 1). Aucune espèce parasite n'est simultanément présente chez plus d'une espèce hôte (Tableau 1). Dans les populations d'hôtes examinés, les prévalences parasitaires varient de 5,2 à 93,1\%. Myxobolus pethericii et $H$. pethericii sont apparues fréquentes $(\operatorname{Pr}>$ 50\%); Myxobolus sp.1, Myxobolus sp.2, M. gariepinus, $M$. africanus, $H$. nkamensis et Myxidium petrocephali sont apparues secondaires $(10 \% \leq \operatorname{Pr} \leq 50 \%)$ alors que $M$. camerounensis et Myxidium sangei sont apparues rares $(\operatorname{Pr}<10 \%)$ (Tableau 1). Myxobolus sp.1, Myxobolus sp.2, M. pethericii, M. gariepinus, M. africanus, $H$. pethericii et $H$. nkamensis sont les seules espèces parasites qui forment des kystes chez leurs hôtes. Pour ces espèces parasites, la charge kystique moyenne s'est avérée élevée ( $\overline{\mathrm{x}}>100$ kystes) pour $H$. pethericii et $H$. nkamensis; moyenne (50 $\leq \overline{\mathrm{x}} \leq 100$ kystes) pour Myxobolus sp.2 ; faible $(10 \leq \overline{\mathrm{x}} \leq 50$ kystes) pour Myxobolus sp.1, M. pethericii, $M$. africanus, et très faible ( $\overline{\mathrm{x}}<10$ kystes) pour M. gariepinus.

\section{Variation du parasitisme en fonction de la} taille du poisson

La proportion de poissons infestés (toutes les espèces parasites présentes confondues) varie selon les classes de taille chez $C$. petherici $\left(\chi^{2}=12,95 ; \operatorname{ddl}=3 ; \mathrm{P}=\right.$ $0,005)$ et $C$. pachynema $\left(\chi^{2}=83,88 ; \mathrm{ddl}=3\right.$; $\mathrm{P}<0,001)$. Chez ces hôtes, le pourcentage d'individus parasités diminue lorsque la taille de l'hôte augmente. Bien que la différence observée ne soit pas significative, les spécimens de $H$. odoe de taille moyenne semblent plus parasités. Une corrélation négative et hautement significative a été enregistrée entre la taille de $C$. petherici et le pourcentage d'infestation par M. pethericii ( $\mathrm{r}$ $=-0,158 ; \mathrm{P}=0,002)$ et par H. pethericii $(\mathrm{r}=$ 0,19; $\mathrm{P}<0,001)$. Par contre, l'occurrence de M. petrocephali est positivement corrélée ( $\mathrm{r}=$ $0,105 ; \mathrm{P}=0,045)$ à la taille de son hôte. Le test de Khi deux montre que les infestations par $M$. pethericii et $H$. pethericii varient significativement entre les différentes classes de taille $\left(\chi^{2}=8,77\right.$; ddl $=3 ; \mathrm{P}=0,032$ pour M. pethericii et $\chi^{2}=18,71 ; \mathrm{ddl}=3 ; \mathrm{P}<0,001$ pour $H$. pethericii). Le pourcentage d'infestation de $M$. petrocephali dans les différentes classes de taille varie entre 6,5 et 15,6 \%. Pour ce Myxidium, l'occurrence augmente progressivement avec la taille de l'hôte (Tableau 2). Le test du Khi deux a révélé une différence significative $\left(\chi^{2}=\right.$ 11,54; ddl $=3 ; \mathrm{P}=0,009)$ dans la distribution de $H$. nkamensis entre les différentes classes de taille (Tableau 3). Par ailleurs, on note une corrélation positive et significative $(r=0,279 ; \mathrm{P}=0,006)$ entre la fréquence d'infestation par cette Myxosporidie et la taille de son hôte. Au cours de cette étude, une corrélation négative et significative a été enregistrée entre la taille de $C$. pachynema et les prévalences de $M$. gariepinus $(\mathrm{r}=-0,346 ; \mathrm{P}<0,001)$ et $M$. nkamensis $(\mathrm{r}=-0,343 ; \mathrm{P}<0,001)$ (Tableau 4).

Chez M. nkamensis, le pourcentage d'infestation varie significativement avec les classes de taille $\left(\chi^{2}=63,94 ;\right.$ ddl $=3 ; \mathrm{P}<$ $0,001)$. La variation de la charge kystique moyenne en fonction des classes de taille est significative pour $M$. pethericii $(\mathrm{H}=24,80$; $\mathrm{ddl}=3 ; \mathrm{P}<0,001)$ et $H$. pethericii $(\mathrm{H}=$ 32,97; ddl = 3; P < 0,001) (Tableau 1). Le coefficient de corrélation entre la charge kystique moyenne de $M$. pethericii et la taille de $C$. petherici est $\mathrm{r}=-0,296, \mathrm{P}<0,001$ alors 
qu'il vaut $\mathrm{r}=-0,190, \mathrm{P}<0,001$ entre la charge kystique de $H$. pethericii et la taille du même hôte. Myxobolus africanus, présente une charge kystique moyenne faible $(10,33 \leq$ $\overline{\mathrm{x}} \leq 18,5$ kystes) dans toutes les classes de taille (Tableau 3). La charge kystique moyenne de Myxobolus sp.2 est élevée ( $\overline{\mathrm{x}}=$ 118 kystes) dans la classe de taille [188223[, moyenne ( $\overline{\mathrm{x}}=82,5$ kystes) dans la classe [118-153[ et faible ( $\overline{\mathrm{x}}=45,5$ kystes) dans la classe de taille [153-188[ (Tableau 3). Les individus de $\mathrm{LS} \geq 223 \mathrm{~mm}$ ne portent pas de kystes de Myxobolus sp.2. Un seul individu porte des kystes à Myxobolus sp.1 dans la classe de taille [118-153[ et chez les individus de LS $\geq 223 \mathrm{~mm}$. Dans les deux autres classes de tailles ([153-188[ et [188-223[), la charge kystique moyenne est inférieure à 11 kystes (Tableau 3). La charge kystique moyenne de H. nkamensis est élevée $(126 \leq \overline{\mathrm{x}} \leq 337,33$ kystes) dans toutes les classes de taille (Tableau 3). Notons qu'un individu mâle de H. odoe (de longueur standard $183 \mathrm{~mm}$ ) a été trouvé porteur de 4200 kystes de $H$. nkamensis.

\section{Variation du parasitisme en fonction du sexe du poisson}

Les pourcentages d'infestation des Myxosporidies étudiées sont statistiquement comparables entre les poissons des deux sexes ( $\mathrm{P}>0,05)$ sauf pour H. pethericii. Cette Henneguya est significativement plus représentée $\left(\chi^{2}=4,066 ; \mathrm{P}=0,044\right)$ chez les Anabantidae femelles $(62,7 \%)$ que chez les mâles (52,1\%) (Tableau 5). L'intensité d'accumulation des kystes de Myxosporidies étudiées est statistiquement la même entre les hôtes mâles et femelles (Tableau 5).
Variation du parasitisme en fonction des organes du poisson

Le nombre d'organes affectés par une espèce parasite varie de 1 à 16 . La branchie constitue l'organe le plus fréquemment affecté par les Myxosporidies étudiées (Tableau 6). Le pourcentage d'infestation varie très significativement selon les organes cibles pour M. pethericii $\left(\chi^{2}=1424,67 ; \mathrm{ddl}=15 ; \mathrm{P}<\right.$ $0,001), H$. pethericii $\left(\chi^{2}=526,65 ; \mathrm{ddl}=3 ; \mathrm{P}\right.$ $<0,001)$, M. africanus $\left(\chi^{2}=14,02 ; \mathrm{ddl}=2 ; \mathrm{P}\right.$ $<0,001)$ et $M$. gariepinus $\left(\chi^{2}=362,73 ; \mathrm{ddl}=\right.$ 7 ; $\mathrm{P}<0,001)$. Bien que colonisant seize organes chez $C$. petherici, $M$. pethericii ne forme de kystes que dans 13 (Tableau 6). La comparaison de la charge kystique moyenne en fonction des organes montre une différence hautement significative pour $M$. pethericii $(\mathrm{H}$ $=104,08 ; \mathrm{ddl}=12 ; \mathrm{P}<0,001)$ et $H$. pethericii $(\mathrm{H}=17,42 ; \mathrm{ddl}=3 ; \mathrm{P}<0,001)$.

\section{Variation saisonnière des indices épidémiologiques}

Evalué en fonction des saisons, le pourcentage d'infestation de M. gariepinus $\left(\chi^{2}\right.$ $=10,63 ; \mathrm{P}<0,001)$, M. petrocephali $\left(\chi^{2}=\right.$ $3,86 ; \mathrm{P}=0,049)$ et $M$. nkamensis $\left(\chi^{2}=29,56\right.$; $\mathrm{P}<0,001)$ est significativement plus élevé en saison pluvieuse qu'en saison sèche. La charge kystique moyenne saisonnière de $M$. pethericii est faible et vaut 30,65 kystes en saison sèche et 28,48 kystes en saison pluvieuse (Figure 2). La différence observée est significative $(\mathrm{U}=9442,5 ; \mathrm{p}=0,013)$. De même, la charge kystique moyenne de $H$. pethericii est significativement $(\mathrm{U}=3967$; $\mathrm{p}$ $=0,013)$ plus importante en saison sèche $(\overline{\mathrm{x}}$ $=186,85$ kystes) qu'en saison pluvieuse $(\overline{\mathrm{x}}=$ 121,88 kystes) (Figure 3). 
G. B. LEKEUFACK FOLEFACK et A. FOMENA / Int. J. Biol. Chem. Sci. 7(6): 2301-2316, 2013

Tableau 1 : Prévalences et charges kystiques moyennes des parasites chez Ctenopoma petherici, Hepsetus odoe et Clarias pachynema.

\begin{tabular}{|c|c|c|c|c|c|c|c|c|}
\hline \multirow[t]{2}{*}{ Espèce parasite } & \multirow[t]{2}{*}{ Espèce hôte } & \multirow{2}{*}{$\begin{array}{c}\text { Nombre } \\
\text { de poisons } \\
\text { examinés }\end{array}$} & \multirow{2}{*}{$\begin{array}{c}\text { Nombre } \\
\text { de poisson } \\
\text { infestés }\end{array}$} & \multirow{2}{*}{$\begin{array}{c}\text { Nombre } \\
\text { de poisons } \\
\text { hébergeant } \\
\text { des kystes }\end{array}$} & \multirow[t]{2}{*}{ Prévalence } & \multicolumn{3}{|c|}{ Nombre de kyste } \\
\hline & & & & & & $\begin{array}{l}\text { Charge } \\
\text { kystique } \\
\text { moyenne }\end{array}$ & $\begin{array}{c}\text { Variation } \\
\text { charge } \\
\text { kystique }\end{array}$ & $\begin{array}{c}\text { Ecart } \\
\text { type }\end{array}$ \\
\hline Myxobolus pethericii $* * *$ & Ctenopoma petherici & 364 & 339 & 307 & 93,1 & 29,35 & $1-941$ & 65,13 \\
\hline Myxobolus gariepinus** & Clarias pachynema & 402 & 146 & 143 & 36,3 & 3,55 & $1-55$ & 5,65 \\
\hline Myxobolus camerounensis* & Clarias pachynema & 402 & 23 & l & 5,7 & l & l & $/ /$ \\
\hline Myxobolus africanus** & Hepsetus odoe & 97 & 34 & 27 & 35,1 & 15,77 & $1-42$ & 13,53 \\
\hline Myxobolus sp.1** & Hepsetus odoe & 97 & 14 & 9 & 14,4 & 25,77 & 1 & 159 \\
\hline Myxobolus sp. $2 * *$ & Hepsetus odoe & 97 & 12 & 12 & 12,4 & 63,75 & $1-220$ & 61,46 \\
\hline Heneguya pethericii*** & Ctenopoma petherci & 364 & 206 & 206 & 56,6 & 146,48 & $1-1465$ & 216,28 \\
\hline Heneguya nkamensis** & Hepsetus odoe & 97 & 43 & 43 & 44,3 & 207,46 & $1-4200$ & 654,89 \\
\hline Myxidium petrocephali** & Ctenopoma petherici & 364 & 37 & l & 10,2 & I & I & // \\
\hline Myxidium nkamensis** & Clarias pachynema & 402 & 104 & l & 25,9 & l & l & $/ /$ \\
\hline Myxidium sangei* & Hepsetus odoe & 97 & 5 & l & 5,2 & l & l & $/ /$ \\
\hline
\end{tabular}


G. B. LEKEUFACK FOLEFACK et A. FOMENA / Int. J. Biol. Chem. Sci. 7(6): 2301-2316, 2013

Tableau 2: Variation du pourcentage d'infestation et de la charge kystique moyenne de Myxobolus pathericii, Heneguya pethericii et Myxidium petrocephali en fonction des classes de taille chez Ctenopoma petherici.

\begin{tabular}{|c|c|c|c|c|c|c|}
\hline \multicolumn{2}{|c|}{ Classe de taille } & \multicolumn{2}{|c|}{ Myxobolus pethericii } & \multicolumn{2}{|c|}{ Henneguya pethericii } & \multirow{2}{*}{$\frac{\text { Myxidium petrocephal }}{\%(n)}$} \\
\hline & $\mathbf{N}$ & $\%(n)$ & $\overline{\mathbf{x}}(\mathbf{s}) \mathbf{n k}$ & $\%(n)$ & $\overline{\mathbf{x}}(\mathbf{s}) \mathbf{n k}$ & \\
\hline$[51-76[$ & 46 & $100(46)$ & $26,48(40,15) 45$ & $78,3(36)$ & $304,11(303,56) 36$ & $6,5(3)$ \\
\hline$[76-101[$ & 70 & $92,9(65)$ & $36,45(44,96) 59$ & $57,1(40)$ & $107,77(128,03) 40$ & $7,1(5)$ \\
\hline$[101-126[$ & 203 & $93,6(190)$ & $31,41(79,97) 171$ & $56,7(115)$ & $127,53(197,56) 115$ & $10,8(22)$ \\
\hline$\geq 126$ & 45 & $84,4(38)$ & $9,31(13,45) 32$ & $33,3(15)$ & $16,66(30,69) 15$ & $15,6(7)$ \\
\hline TOTAL & 364 & 93,1(339) & $29,35(65,13) 307$ & $56,6(206)$ & $146,48(216,28) 206$ & $10,2(37)$ \\
\hline
\end{tabular}

$\mathrm{N}$ : nombre de poissons examinés; n: nombre de poissons parasités; nk: nombre de poissons hébergeant au moins un kyste ; $\bar{x}$ : charge kystique moyenne :

$\mathrm{s}$ : écart type ; \% : pourcentage d'infestation.

Tableau 3: Variation du pourcentage d'infestation et de la charge kystique moyenne de Myxobolus africanus, Myxobolus sp.1, Myxobolus sp.2 et Heneguya nkamensis en fonction des classes de taille chez Hepsetus odoe.

\begin{tabular}{|c|c|c|c|c|c|c|c|c|c|}
\hline \multicolumn{2}{|c|}{ Classe de taille } & \multicolumn{2}{|c|}{ Myxobolus africanus } & \multicolumn{2}{|c|}{ Myxobolus sp.1 } & \multicolumn{2}{|c|}{ Myxobolus sp.2 } & \multicolumn{2}{|c|}{ Henneguya nkamensis } \\
\hline & $\mathbf{N}$ & $\%(n)$ & $\overline{\mathbf{x}}(\mathbf{s}) \mathbf{n k}$ & $\%(n)$ & $\overline{\mathbf{x}}(\mathbf{s}) \mathbf{n k}$ & $\%(n)$ & $\overline{\mathbf{x}}(\mathbf{s}) \mathbf{n k}$ & $\%(n)$ & $\overline{\mathbf{x}}(\mathbf{s}) \mathbf{n k}$ \\
\hline$[118-153[$ & 24 & $33,3(8)$ & $15,25(13,86) 6$ & $8,3(2)$ & $14(/ /) 1$ & $8,3(2)$ & $82,5(13,43) 2$ & $20,8(5)$ & $177,4(245,97) 5$ \\
\hline$[153-188[$ & 36 & $41,7(15)$ & $18,5(15,57) 12$ & $19,4(7)$ & $5,66(6,35) 3$ & $22,2(8)$ & $45,5(40,19) 8$ & $41,7(15)$ & $337,33(1070,42) 15$ \\
\hline$[188-223[$ & 30 & $26,7(8)$ & $10,33(9,6) 6$ & $13,3(4)$ & $10,5(17,01) 4$ & $6,7(2)$ & $118(144,24) 2$ & $66,7(20)$ & $129(263,93) 20$ \\
\hline$\geq 223$ & 7 & $42,9(3)$ & $20(/ /) 1$ & $14,3(1)$ & $159(/ /) 1$ & 1 & 1 & $42,9(3)$ & $126(65) 3$ \\
\hline TOTAL & 97 & $35,1(34)$ & $15,77(13,53) 27$ & $14,4(14)$ & $25,77(51,21) 9$ & $12,4(12)$ & $63,75(61,46) 12$ & $44,3(43)$ & $207,46(654,89) 43$ \\
\hline
\end{tabular}

N: nombre de poissons examinés; n: nombre de poissons parasités; nk: nombre de poissons hébergeant au moins un kyste ; $\overline{\mathrm{x}}$ : charge kystique moyenne ;

$\mathrm{s}$ : écart type ; \% : pourcentage d'infestation ; / : aucun poisson parasité ; // : aucune valeur. 
Tableau 4: Variation du pourcentage d'infestation et de la charge kystique moyenne de Myxobolus gariepinus, et Myxidium nkamensis en fonction des classes de taille chez Clarias pachynema.

\begin{tabular}{|c|c|c|c|c|}
\hline \multicolumn{2}{|c|}{ Classe de taille } & \multicolumn{2}{|c|}{ Myxobolus gariepinus } & \multirow{2}{*}{$\begin{array}{c}\text { Myxidium nkamensis } \\
\%(\mathbf{n})\end{array}$} \\
\hline & $\mathbf{N}$ & $\%(n)$ & $\bar{X}(\mathbf{s}) \mathbf{n k}$ & \\
\hline [55-105[ & 216 & $76(111)$ & $3,96(6,33) 109$ & $40,7(88)$ \\
\hline$[105-155[$ & 39 & $8,9(13)$ & $2,58(2,06) 12$ & $28,2(11)$ \\
\hline$[155-205[$ & 80 & $10,3(15)$ & $2,4(2,26) 15$ & $5(4)$ \\
\hline$\geq 205$ & 67 & $4,8(7)$ & $1,28(0,48) 7$ & $1,5(1)$ \\
\hline TOTAL & 402 & $36,3(146)$ & $3,55(5,65) 143$ & $25,9(104)$ \\
\hline
\end{tabular}

$\mathrm{N}$ : nombre de poissons examinés; n: nombre de poissons parasités; nk: nombre de poissons hébergeant au moins un kyste ; $\overline{\mathrm{x}}$ : charge kystique moyenne ; $\mathrm{s}$ : écart type ; \% : pourcentage d'infestation.

Tableau 5: Variation du pourcentage d'infestation et de la charge kystique moyenne des différentes espèces de Myxosporidies étudiées en fonction du sexe.

\begin{tabular}{|c|c|c|c|c|c|}
\hline \multirow{3}{*}{ Espèce hôte } & \multirow{3}{*}{ Espèce parasite } & \multicolumn{4}{|c|}{ Sexe de l'hôte } \\
\hline & & \multicolumn{2}{|r|}{ Mâle } & \multicolumn{2}{|c|}{ Femelle } \\
\hline & & $\%(\mathbf{n})$ & $\bar{x}(s) n c$ & $\%(n)$ & $\bar{x}(\mathrm{~s}) \mathrm{nc}$ \\
\hline Ctenopoma & Myxobolus pethericii & $92,4(195)$ & $27,17(42,81) 174$ & $94,1(144)$ & $32,22(86,12) 133$ \\
\hline petherici & Henneguya pethericii & $52,1(110)$ & $162,31(239,12) 110$ & $62,7 *(96)$ & $128,35(186,33) 96$ \\
\hline$N=364$ & Myxidium petrocephali & $9(19)$ & l & $11,8(18)$ & l \\
\hline $\begin{array}{l}\hat{O}=\mathbf{2 1 1} \\
q=\mathbf{1 5 3}\end{array}$ & Total & $94,3(199)$ & & $95,4(146)$ & \\
\hline Hepsetus & Myxobolus africanus & $41,3(26)$ & $16,57(13,56) 21$ & $23,5(8)$ & $13(14,28) 6$ \\
\hline odoe & Myxobolus sp.1 & $15,9(10)$ & $9,12(12,05) 8$ & $11,8(4)$ & $159(00) 1$ \\
\hline $\mathbf{N}=97$ & Myxobolus sp.2 & $12,7(8)$ & $79,12(66,78) 8$ & $11,8(4)$ & $33(39,47) 4$ \\
\hline$\widehat{\partial}=63$ & Henneguya nkamensis & $41,3(26)$ & $245,46(815,65) 26$ & $50(17)$ & $149,35(283,49) 17$ \\
\hline$q=34$ & Total & $65,1(41)$ & & $58,8(20)$ & \\
\hline Clarias & Myxobolus gariepinus & $33,2(69)$ & $2,65(2,49) 67$ & $39,7(77)$ & $4,34(7,33) 76$ \\
\hline pachynema & Myxidium nkamensis & $25,5(53)$ & l & $26,3(51)$ & l \\
\hline$N=402$ & & & & & \\
\hline$\hat{O}=\mathbf{2 0 8}$ & Total & $46,2(96)$ & & $53,1(103)$ & \\
\hline$q=\mathbf{1 9 4}$ & & & & & \\
\hline
\end{tabular}

$\mathrm{N}$ : nombre de poissons examinés; n: nombre de poissons parasité; nk: nombre de poissons hébergeant au moins un kyste; $\widehat{\delta}$ : nombre de poissons mâle; $ᄋ$ : nombre de poissons femelle ; $\bar{x}$ : charge kystique moyenne; S: écart type; \%: pourcentage d'infestation; *: significativement différent; /: pas de kyste. 
G. B. LEKEUFACK FOLEFACK et A. FOMENA / Int. J. Biol. Chem. Sci. 7(6): 2301-2316, 2013

Tableau 6: Variation du pourcentage d'infestation et de la charge kystique moyenne des différentes espèces de Myxosporidies étudiées en fonction des organes.

\begin{tabular}{|c|c|c|c|c|c|c|c|c|c|c|c|c|c|c|c|c|c|c|c|}
\hline \multirow[b]{2}{*}{ Espèce parasite } & \multicolumn{19}{|c|}{ Organe } \\
\hline & & 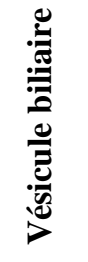 & 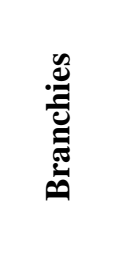 & 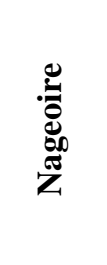 & 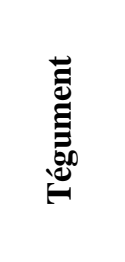 & $\frac{\tilde{U}}{\tilde{E}}$ & $\stackrel{\mathscr{0}}{\mathscr{\theta}}$ & $\stackrel{\mathscr{e}}{\ddot{e}}$ & 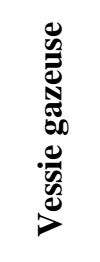 & 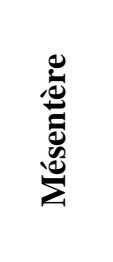 & 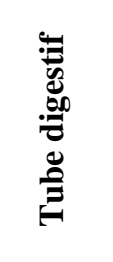 & 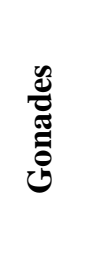 & 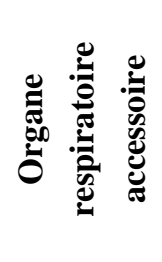 & $\stackrel{\circlearrowright}{\partial}$ & $\underset{\mathscr{D}}{\stackrel{\mathscr{T}}{*}}$ & 己َd & 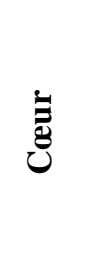 & & 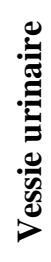 \\
\hline \multirow{3}{*}{$\begin{array}{c}\text { Myxobolus } \\
\text { pethericii }\end{array}$} & $\%$ & 4,1 & 67,3 & 7,7 & 3 & 19,2 & 45,6 & 36,8 & 18,7 & 17 & 12,9 & 12,9 & 5,2 & 3,8 & 1,4 & 1,1 & 0,8 & & \\
\hline & $\overline{\mathrm{x}}$ & & 14,06 & 9,14 & 102,63 & 10,54 & 18,65 & & 9,95 & 16,98 & 16,36 & 7 & 2,63 & 3,14 & & 4 & 5,33 & - & - \\
\hline & $\mathrm{s}$ & & 23,63 & 17,74 & 196,46 & 28,49 & 45,61 & & 14,02 & 26,8 & 18 & 8,64 & 3,83 & 3,61 & & 1,41 & 4,5 & & \\
\hline \multirow{3}{*}{$\begin{array}{c}\text { Henneguya } \\
\text { pethericii }\end{array}$} & $\%$ & & 55,2 & 4,1 & 4,9 & 1,9 & & & & & & & & & & & & & \\
\hline & $\overline{\mathrm{x}}$ & - & 141,82 & 30,06 & 63,72 & 10,28 & - & - & - & - & - & - & - & - & - & - & - & - & - \\
\hline & $\mathrm{s}$ & & 216,39 & 49,19 & 98,32 & 14,1 & & & & & & & & & & & & & \\
\hline $\begin{array}{c}\text { Myxidium } \\
\text { petrocephali }\end{array}$ & $\%$ & 10,2 & - & - & - & - & - & - & - & - & - & - & - & - & - & - & - & - & - \\
\hline \multirow{3}{*}{$\begin{array}{c}\text { Myxobolus } \\
\text { africanus }\end{array}$} & $\%$ & & 24,7 & 7,2 & & & & 10,3 & & & & & & & & & & & \\
\hline & $\overline{\mathrm{x}}$ & - & 14,29 & 11,86 & - & - & - & & - & - & - & - & - & - & - & - & - & - & - \\
\hline & $\mathrm{s}$ & & 12,84 & 11,34 & & & & & & & & & & & & & & & \\
\hline \multirow{3}{*}{ Myxobolus sp.1 } & $\%$ & & 9,3 & & & & & 8,2 & & & & & & & & & & & \\
\hline & $\overline{\mathrm{x}}$ & - & 25,88 & - & - & - & - & & - & - & - & - & - & - & - & - & - & - & - \\
\hline & $\mathrm{s}$ & & 51,16 & & & & & & & & & & & & & & & & \\
\hline
\end{tabular}


G. B. LEKEUFACK FOLEFACK et A. FOMENA / Int. J. Biol. Chem. Sci. 7(6): 2301-2316, 2013

\begin{tabular}{|c|c|c|c|c|c|c|c|c|c|c|c|c|c|c|c|c|c|c|c|}
\hline \multirow{3}{*}{ Myxobolus sp.2 } & $\%$ & & & & 12,4 & & & & & & & & & & & & & & \\
\hline & $\overline{\mathrm{x}}$ & - & - & - & 63,95 & - & - & - & - & - & - & - & - & - & - & - & - & - & - \\
\hline & $\mathrm{s}$ & & & & 61,46 & & & & & & & & & & & & & & \\
\hline \multirow{3}{*}{$\begin{array}{c}\text { Henneguya } \\
\text { nkamensis }\end{array}$} & $\%$ & & 44,3 & & & & & & & & & & & & & & & & \\
\hline & $\overline{\mathrm{x}}$ & - & 207,46 & - & - & - & - & - & - & - & - & - & - & - & - & - & - & - & - \\
\hline & $\mathrm{s}$ & & 654,89 & & & & & & & & & & & & & & & & \\
\hline \multirow{3}{*}{$\begin{array}{l}\text { Myxobolus } \\
\text { gariepinus }\end{array}$} & $\%$ & 0,5 & & 8,2 & 2 & & & 4 & & 7,5 & & 3 & & & & & & 27,6 & 0,5 \\
\hline & $\overline{\mathrm{x}}$ & & - & 5,97 & 3,75 & - & - & & - & 2,06 & - & 1,75 & - & - & - & - & - & 1,75 & \\
\hline & $\mathrm{s}$ & & & 9,14 & 4,65 & & & & & 1,48 & & 0,96 & & & & & & 1,13 & \\
\hline $\begin{array}{l}\text { Myxidium } \\
\text { nkamensis }\end{array}$ & $\%$ & 25,9 & - & - & - & - & - & - & - & - & - & - & - & - & - & - & - & - & - \\
\hline
\end{tabular}



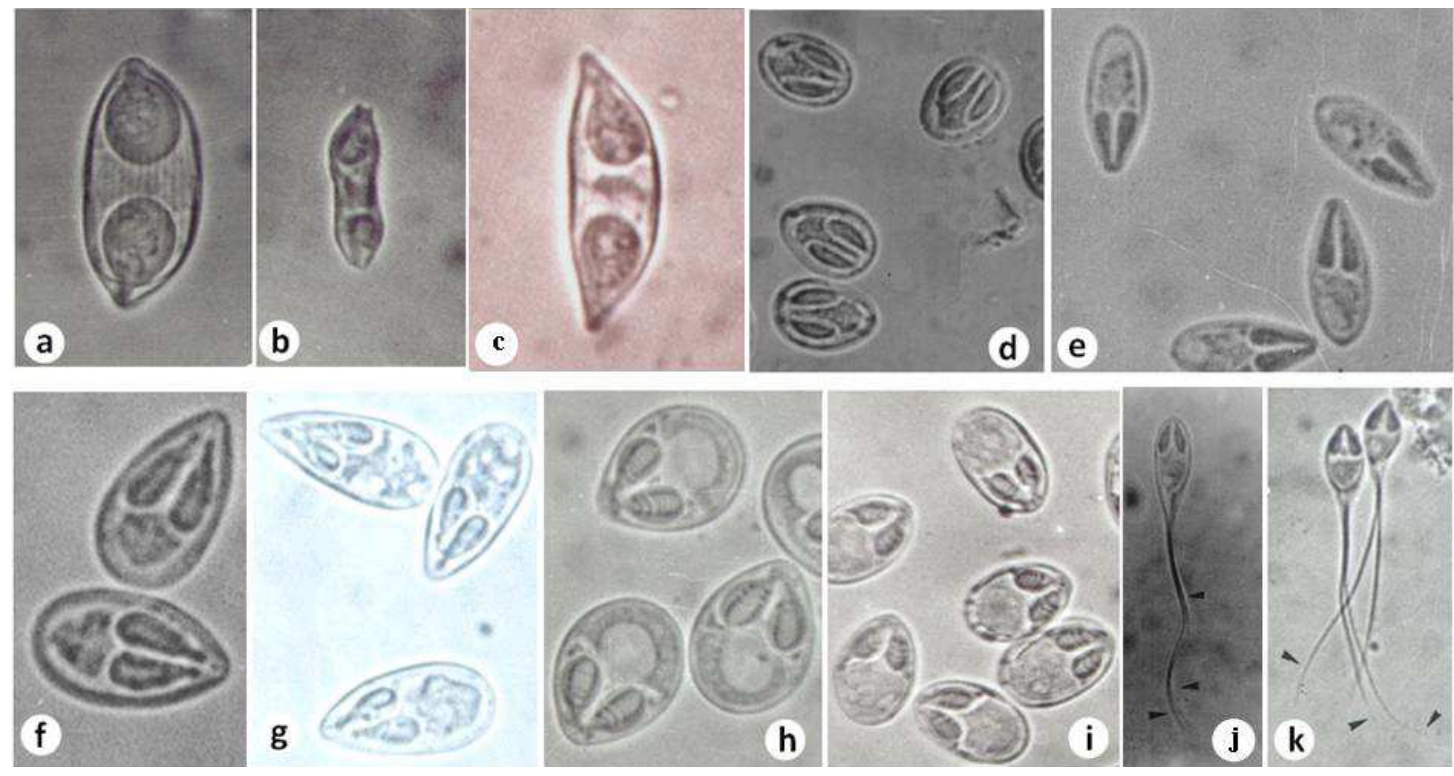

a: Myxidium nkamensis FOMENA, LEKEUFACK FOLEFACK \& BOUIX, 2010 (x 1100);

b: Myxidium sangei FOMENA, LEKEUFACK FOLEFACK \& BOUIX, 2010 (x 1400);

c: Myxidium petrocephali FOMENA \& BOUIX, 1986 (x 1415);

d: Myxobolus pethericii FOMENA, LEKEUFACK FOLEFACK \& TANG II, 2007 (x 900);

e: Myxobolus sp.1 (x 1000);

f: Myxobolus sp.2 (x 1900);

g: M. africanus FOMENA, BOUIX \& BIRGI, 1985 (x 1200);

h: M. camerounensis FOMENA, MARQUES \& BOUIX, 1993 (x 1100);

i: M. gariepinus REED, BASSON \& VAN AS, 2003 (x 800);

j: Henneguya pethericii FOMENA, LEKEUFACK FOLEFACK \& BOUIX, 2008 (x 760);

k: H. nkamensis FOMENA, LEKEUFACK FOLEFACK \& BOUIX, 2008 (x 900).

Figure 1: Photographies des spores des différentes espèces de Myxosporidies étudiées.

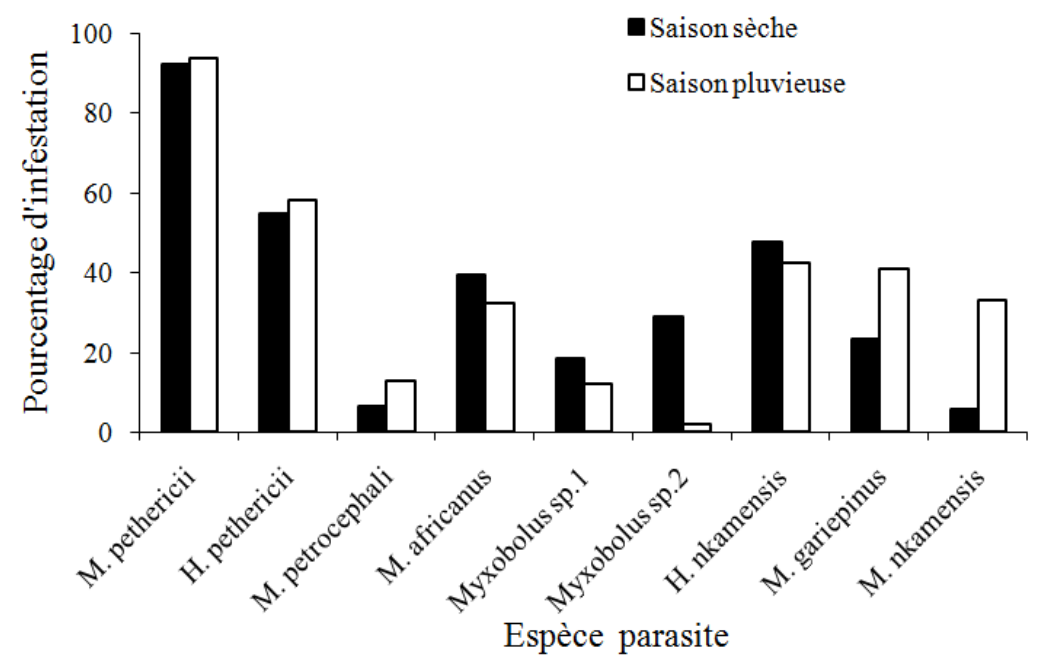

Figure 2: Variation du pourcentage d'infestation parasitaire en fonction des saisons. 


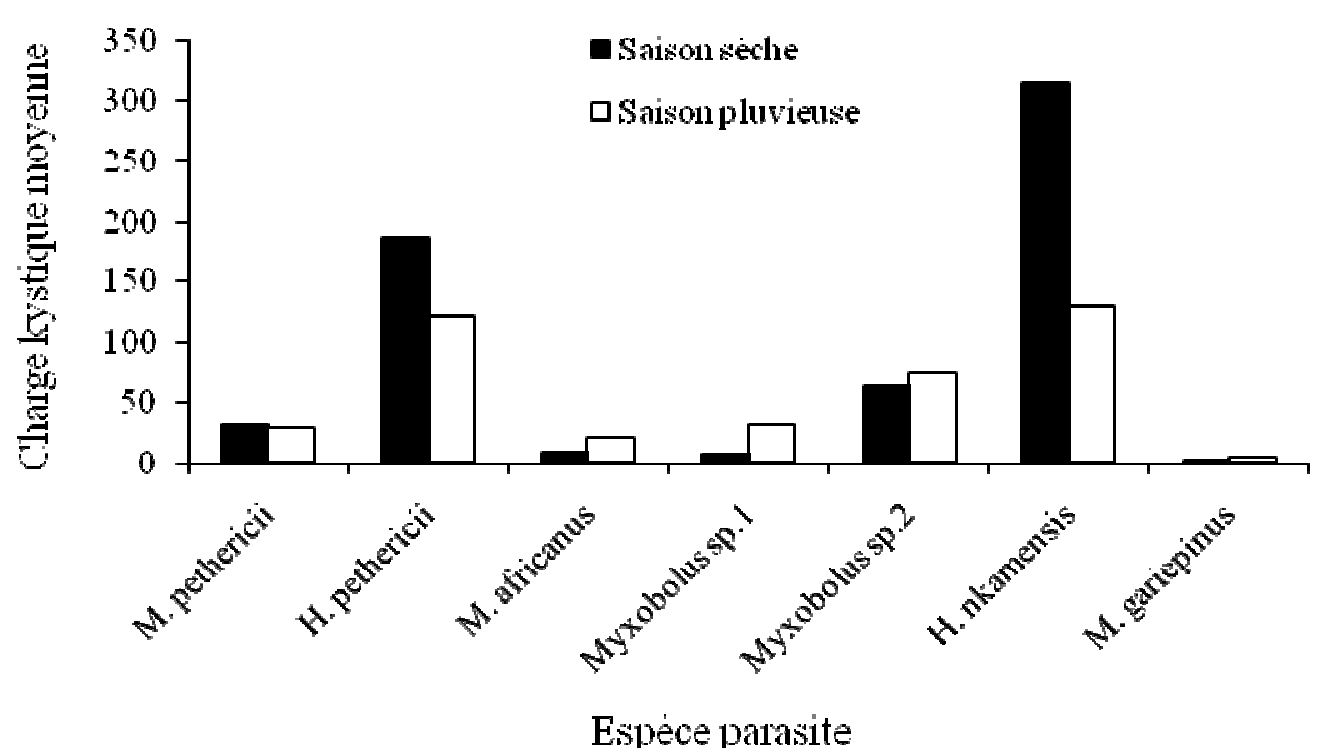

Figure 3: Variation de la charge kystique moyenne en fonction des saisons.

\section{DISCUSSION}

La majorité des poissons hôtes échantillonnés au cours de ce travail sont jeunes car les longueurs standards maximales connues pour ces derniers sont de $180 \mathrm{~mm}$ pour $C$. petherici, $445 \mathrm{~mm}$ pour $H$. odoe et $356 \mathrm{~mm}$ pour C. pachynema (Stiassny et al., 2007). Ceci peut s'expliqué par la technique de pêche utilisée, technique qui n'était pas appropriée pour la récolte des poissons de grande taille. Cependant, l'on peut penser que $\mathrm{du}$ fait du parasitisme, plusieurs jeunes poissons ne parviennent pas au stade adulte.

D'après Konan et al. (2013) le rapport des sexes est généralement voisin de 1 , mais des écarts par rapport à cette situation existent pour différentes raisons possibles : croissance différentielle en fonction des sexes, capture différente des individus des deux sexes selon les techniques de pêche, mortalité affectant les sexes de manière différente au cours de leur vie, comportement grégaire des poissons fortement lié au sexe.

Etant donnée l'implication des Oligochètes dans le cycle de développement de plusieurs espèces de Myxosporidies (Kent et al., 2001; Lom et Dyková, 2006), la variation du degré de parasitisme observée chez les différents hôtes peut être attribuée à la proximité des poissons (hôtes intermédiaires) et des Oligochètes (hôtes définitifs). Cette variation n'impliquerait aucun effet de compétition entre les espèces parasites car Sitja-Bobadilla (2008) signalent l'absence de compétition inter et intraspécifiques chez les Myxosporidies. Selon ce même auteur, cette absence de compétition favoriserait le polyparasitisme chez les hôtes. Les indices épidémiologiques élevés (prévalence et charge kystique moyenne) observés chez $C$. petherici s'expliqueraient par les caractéristiques intrinsèques de cet hôte qui le prédisposent aux infestations. Ces poissons sont des microprédateurs se nourrissant sur de Oligochètes (Randle et Chapman, 2004), ce qui les rend plus vulnérables par rapport à $H$. odoe et $C$. pachynema qui sont omnivores. En raison de leur rareté, les espèces satellites $(M$. camerounensis et Myxidium sangei) ont été exclues des analyses statistiques car selon Combes (1995), de telles espèces ne sont pas structurantes. Les faibles prévalences de $M$. camerounensis et $M$. sangei trouvées respectivement chez C. pachynema et $H$. odoe laissent penser que ces poissons seraient des hôtes accidentels pour ces parasites. Ces Myxosporidies rares ou satellites retrouvées 
chez ces hôtes peuvent apparaître plus fréquemment chez d'autres espèces hôtes. C'est la cas pour M. camerounensis qui a été décrite pour la première fois chez Oreochromis niloticus au Cameroun (Fomena et al., 1993), puis en 2000, Fall et al. signalent sa présence chez deux Cichlidés (Sarotherodon melanotheron et Tilapia zillii) au Sénégal. En 2002, Reed et al. la retrouvent chez $O$. andersonii et Tilapia ruweti au Botswana. De par la faible prévalence $(5,7 \%)$ obtenue au cours de notre étude, cette Myxosporidie (parasite systémique) autrefois inféodée aux poissons Cichlidae, se serait accidentellement retrouvée chez le Clariidae étudié.

La distribution des Myxosporidies au sein des classes de taille des poissons a montré, en accord avec Viozzi et Flores (2003), Tombi et Bilong Bilong (2004), Abakar-Ousman (2006), Nchoutpouen et al. (2011), l'existence d'une hétérogénéité dans la colonisation de l'hôte. De plus, les plus jeunes spécimens observés dans la rivière Sangé sont plus fréquemment et plus abondamment parasités que les adultes par $M$. pethericii, M. gariepinus, $H$. pethericii et $M$. nkamensis. Cette observation n'est pas originale chez les Myxosporidies car selon Longshaw et al. (2005), les plus jeunes poissons sont plus susceptibles aux Myxosporidies et certaines infestations diminuent avec l'âge de l'hôte. Dans leurs travaux sur plusieurs espèces appartenant aux genres Myxobolus et Myxidium, Longshaw et al. (2005) expliquent la baisse de la prévalence avec l'âge par la réponse immunitaire qui augmente avec l'âge ou la taille de l'hôte. Abakar Ousman (2006) pense également que les fortes fréquences (et probablement les charges kystiques moyenne) des Myxosporidies branchiales semblent caractériser le parasitisme des jeunes Téléostéens. La croissance rapide de l'épithélium branchial dans cette tranche d'âge favoriserait le développement des kystes de Myxosporidies. De plus, Longshaw et al. (2005) pensent que le vieillissement et la rupture des kystes parasitaires surviennent avec l'augmentation de l'âge des poissons hôtes. Notons cependant que M. africanus a été plus fréquente chez les hôtes de taille moyenne pendant que $H$. nkamensis et $M$. petrocephali le sont chez les hôtes de grande taille. Pampoulie et al. (2000) suggèrent que cela serait dû à un effet de cumul des parasites dans les organes des poissons hôtes avec l'âge. Par ailleurs, ces mêmes auteurs pensent que les parasites localisés dans les organes internes ne libèrent leurs différentes formes infestantes qu'après la mort des hôtes. Nos résultats corroborent ceux de Viozzi et Flores (2003) en Argentine car ces auteurs trouvent une corrélation positive et significative entre la prévalence de Myxidium biliare et la taille de son hôte (Galaxia maculatus). Les corrélations négatives et significatives enregistrées entre la taille des hôtes et les charges kystiques moyennes de $M$. pethericii, $M$. gariepinus et $H$. pethericii sont similaires aux observations de Tombi et Bilong Bilong (2004) qui ont noté que les jeunes Barbus martorelli hébergent plus de kystes de Myxosporidies que ceux de grande taille.

Selon Simkova et al. (2005), l'effet du sexe de l'hôte sur la prévalence et la charge kystique moyenne est un phénomène pas très clair. Pour $H$. pethericii, les individus femelles sont plus réceptifs que les mâles, ceci serait dû à la différence du statut physiologique et de la quantité de mucus produit entre les spécimens mâles et femelles.

Le spectre d'organes colonisés par les Myxosporidies étudiées varie d'une espèce parasite à l'autre. Ibrahim et Soliman (2010) pensent que l'hétérogénéité des biotopes crée une série de microenvironnements (sites) distincts qui sont autant d'options d'habitat pour les espèces parasites. La fréquence d'infestation du tégument, des branchies et des nageoires serait dû au fait que ces organes sont en contact direct avec l'environnement aquatique du poisson, offrant ainsi une surface d'attache aux actinospores. Le spectre élevé d'organes cibles observé pour $M$. pethericii (16 organes parasités) et $M$. gariepinus (8 organes) serait dû à leur flexibilité pour les ressources alimentaires. Dans une infection massive des branchies par des kystes de $M$. pethericii, Myxobolus sp.2, M. africanus, $H$. pethericii et $H$. nkamensis, en réduisant la surface épithéliale et en comprimant les 
capillaires sanguins, ces parasites peuvent partiellement compromettre les fonctions de la branchie (Fomena et al., 2008). De plus, la rupture de ces kystes entraîne des inflammations et des hémorragies responsables de l'anémie chez les hôtes. La présence de $M$. pethericii dans les gonades peut avoir des conséquences néfastes sur la pérennité de $C$. petherici dans la rivière Sangé, car il a été démontré que les Myxosporidies se développant dans les gonades de poisson affecte le taux de reproduction de ce dernier soit en détruisant le tissu germinatif soit en se nourrissant sur les spermatozoïdes (Sitja-Bobadilla, 2009).

La saisonnalité influence la prévalence (chez les espèces Myxobolus sp.2, M. gariepinus, Myxidium petrocephali et Myxidium nkamensis) et les charges parasitaires (chez $M$. pethericii et $H$. pethericii). Ceci laisse supposer que la variation des conditions bio-physiques de l'environnement due à la saisonnalité aurait un rôle important sur le parasitisme par les Myxosporidies chez les poissons. L'influence des saisons a été étudiée chez plusieurs espèces de Myxosporidies. On remarque que certains parasites sont présents chez les poissons hôtes pendant toute l'année (Tombi, 2005; Abakar-Ousman, 2006); d'autres présentent une seule apparition, alors que d'autres présentent deux ou trois pics d'infestation chaque année (Blazer et al., 2003). Etant donnée l'implication des Oligochètes dans le cycle de développement des Myxosporidies (Markiw et Wolf, 1983), cette variation saisonnière serait due au taux de libération des Actinospores par les Oligochètes, taux qui varie en fonction des saisons (Özer et al., 2002). Pampoulie et al. (2000) pensent que la saison sèche serait favorable à l'installation du parasitisme par les Myxosporidies. Par ailleurs, Gbankoto et al. (2001) signalent une saisonnalité sur la prévalence de Myxobolus sp. et M. zillii (parasites des branchies de Tilapia zillii et de Sarotherodon melanotheron au Bénin). Les prévalences de ces deux espèces sont plus fortes en saison sèche (Gbankoto et al., 2001). Au cours de notre étude, des observations similaires ont été faites pour Myxobolus sp.2.
Les résultats enregistrés sur $M$. gariepinus, $M$. nkamensis et $M$. petrocephali corroborent ceux obtenus par Abakar-Ousman (2006) au Tchad. Cet auteur remarque que l'occurrence de Myxobolus agolus, M. brachysporus, $M$. clarii, M. tilapiae, M. cichlidarum et $M$. heterosporus dans le fleuve Chari est plus élevée pendant la saison de pluies. Il conclut que cela est certainement dû à une pollution moins forte du cours d'eau en cette saison.

\section{REFERENCES}

Abakar O. 2006. Les Myxospories (Myxozoa: Myxosporea) parasites des poissons d'eau douce du Tchad : faunistique et biologie des espèces inféodées à Oreochromis niloticus (Linné, 1758) et Sarotherodon galilaeus (Linné, 1758) Cichlidae. Thèse de Doctorat/PhD, Université de Yaoundé I, Yaoundé, p. 163.

Abakar O, Bilong CF, Njine T, Fomena A. 2007. Structure and dynamics of myxosporean parasites component communities in two fresh water Cichlids in the Chari River (Republic of Chad). Pak. J. Biol. Sci., 10: 692-700.

Bilong Bilong CF, Njine T. 1998. Dynamique des populations de trois monogènes parasites d'Hemichromis fasciatus PETERS, 1858 dans le lac municipal de Yaoundé, et intérêt possible en pisciculture intensive. Ann. Fac. Sci. Univ. Yaoundé I., Sér. Sci. Nat. Vie, 34: 295 - 303.

Blazer VS, Waldrop TB, Schill WB, Ensmore CL, Smith D. 2003. Effects of water temperature and substrate type on spore production and release in eastern Tubifex tubifex worms infected with Myxobolus cerebralis. J. Parasitol., 89: 21-26.

Boungou M, Sinaré Y, Mano K, Kabré GB. 2013. Parasitic Copepods (Arthropoda, Crustacea, Copepoda) from Fishes in Burkina Faso, Africa. Int. J. Fish. Aquat. Sci., 2: 58-64.

Bush AO, Lafferty KD, Lotz JM, Shostak AW. 1997. Parasitology meets ecology on its own terms. J. Parasitol., 83: 575-583.

Combes C. 1995. Interactions Durables. Ecologie et Evolution du Parasitisme. 
Collection d'Ecologie ( ${ }^{\circ}$ 26). Ed. Masson: Paris.

Fall M, Fomena A, Kostoïngue B, Diebakate C, Faye N, Toguebaye BS. 2000. Myxosporidies (Myxozoa, Myxosporea) parasites des poissons Cichlidae du Cameroun, du Sénégal et du Tchad, avec la description de deux nouvelles espèces. Ann. Sci. Nat., 21: 81-92.

Fomena A, Marques A, Bouix G. 1993. Myxosporidea (Myxozoa) of Oreochromis niloticus (LINNAEUS, 1757) (Teleost, Cichlidae) in fish-farming pools at Melen (Yaounde, Cameroon, central Africa). J. Afr. Zool., 197: 45 - 56.

Fomena A, Lekeufack Folefack GB, Bouix G. 2008. Three new species of Henneguya (Myxozoa: Myxosporea), parasites of fresh water fishes in Cameroon (central Africa). J. Afrotrop. Zool., 4: 93 - 103.

Gbankoto A, Pampoulie C, Marques A, Sakiti GN. 2001. Occurrence of Myxosporean parasites in the gills of two tilapia species from Lake Nokoué (Bénin, West Africa): effect of host size and sex, and seasonal patterns of infection. Dis. Aquat. Organ, 44: $217-222$.

Ibrahim MM, Soliman MFM. 2010. Prevalence and site prevalence and site preferences of Heterophyid Metacercariae in Tilapia zilli from Ismalia fresh water canal, Egypt. Parasite, 17: 233-239.

Kent ML, Andree KB, Bartholomew KL, ElMatbouli, Desser SS, Delvin RH, Feist SW, Hedrick RP, Hoffman RW, Khattra J, Hallet SL, Lester JG, Longshaw M, Palenzeula O, Siddal ME, Xiao C. 2001. Recent Advances in our knowledge of the Myxozoa. J. Eukaryot. Microbiol., 48: 395-413.

Konan YA, Ouattara S, Kone T, Bamba M, Kone I. 2013. Caractéristiques de la reproduction de Thysochromis ansorgii (Pisces, Cichlidae) dans la forêt des marais Tanoé-Ehy (Côte d'Ivoire). J. Appl. Biosci., 71: 5715-5727.

Lom J, Arthur JR. 1989. A guideline for the preparation of species descriptions in Myxosporea. J. Fish Dis., 12: 151-156.

Lom J, Dyková I. 2006. Myxozoan genera: definition and notes on taxonomy, life- cycle terminology and pathogenic species. Folia Parasitol., 53: 1-36.

Longshaw M, Frear PA, Feist SW. 2005. Description, development and pathogenicity of Myxozoan (Myxozoa: Myxosporea) parasites of juvenile Cyprinids (Pisces: Cyprinidae). J. Fish Dis., 28: 489 - 508.

Markiw ME, Wolf K. 1983. Myxosoma cerebralis (Myxozoa: Myxosporea) etiologic agent of salmonid whirling disease requires tubificid worm (Annelida: Oligochaeta) in its life cycle. J. Protozool., 30: $561-564$.

Mladineo I. 2003. Myxosporidean infections in Adriatic cage-reared fish. Bull. Eur. Assoc. Fish Pathol., 23: 113-123.

Nchoutpouen E, Lekeufack Folefack GB, Fomena A. 2011. Structure and population dynamics of Myxobolus infections in wild and cultured Oreochromis niloticus Linnaeus, 1758 in the Noun division (West-Cameroon). J. Cell Anim. Biol., 5: 254-264.

Özer A, Wootten R, Shinn AP. 2002. Survey of Actinosporean types (Myxozoa) belonging to seven collective groups found in a freshwater salmon farm in Northern Scotland. Folia Parasitol., 49: 189 - 210.

Pampoulie C, Lambert A, Rosecchi E, Bouchereau JL, Crivelli AJ, Morand S. 2000. Host death: a necessary condition for the transmission of Aphalloïdes coelomicola Dollfus, Chabaud, Golvan, 1957. J. Parasitol., 86: 416 - 417.

Piasecki W, Goodwin AE, Eiras JC, Nowak BF. 2004. Importance of Copepoda in freshwater aquaculture. Zoolog. Studies, 43: 193-205.

Randle AM, Chapman LJ. 2004. Habitat use by the African anabantid fish Ctenopoma muriei: implications for costs of air breathing. Ecology of Freshwater Fish, 13: $37-45$.

Reed CC, Basson L, Van As LL. 2002. Myxobulus species (Myxozoa), parasites of fishes in the Okovango River and Delta, Botswana, including description of two new species. Folia Parasitol., 49: 81- 88.

Renault T, Guichard B. 2007. Facteurs de risque d'apparition et d'émergence des 
maladies infectieuses en aquaculture. INRA Prod. Anim., 20: 219-222.

Rioul O. 2008. Théorie des Probabilités. Lavoisier : Paris.

Simkova A, Jarkovsky J, Koubkova B, Barus V, Prokes M. 2005. Associations between fish reproductive cycle and the dynamics of Metazoan parasite infection. Parasitol Res., 95: 65-72.

Sitjà-Bobadilla A. 2008. Fish immune response to Myxozoan parasites. Parasite, 15: 420-425.

Sitjà-Bobadilla A. 2009. Can Myxosporean parasites compromise fish and amphibian reproduction? Proc. R. Soc. B., 276: 28612870.

Stiassny MLG, Teugels GG, Hopkins CD. 2007. Poissons d'Eaux Douces et Saumâtres de la Basse Guinée, Ouest de l'Afrique Centrale (vol 1). Collection Faune et Flore Tropicales: Paris.

Tombi J. 2005. Monogènes et Myxosporidies ectoparasites de Barbus martorelli
(Pisces: $\quad$ Cyprinidae): $\quad$ Structure temporelle des infracommunautés en milieu forestier secondaire et impact potentiel sur la santé des hôtes. Thèse de Doctorat/PhD, Université de Yaoundé I, Yaoundé, p. 135.

Tombi J, Bilong Bilong CF. 2004. Distribution of gill parasites of the freshwater fish Barbus martorelli Roman, 1971 (Teleostei: Cyprinidae) and tendency to inverse intensity evolution between Myxosporidia and Monogenea as a function of the host age. Revue Élev. Méd. Vét. Pays Trop., 57: $71-76$.

Viozzi G, Flores V. 2003. Myxidium biliare sp.n. (Myxozoa) from gall bladder of Galaxias maculatus (Osmeriformes: Galaxidae) in Patagonia (Argentina). Folia Parasitol., 50: 190-194. 